OPEN ACCESS

Edited by:

Guan Zhu,

Texas A\&M University,

United States

Reviewed by:

Jan Mead,

Emory University, United States Alejandro Castellanos-Gonzalez,

The University of Texas Medical

Branch at Galveston, United States

*Correspondence:

Yaoyu Feng

yyfeng@ecust.edu.cn

Lihua Xiao

Ixiao1961@gmail.com

Specialty section:

This article was submitted to

Infectious Diseases,

a section of the journal

Frontiers in Microbiology

Received: 18 March 2019

Accepted: 08 May 2019

Published: 29 May 2019

Citation:

Su J, Jin C, Wu H, Fei J, Li N, Guo Y,

Feng $Y$ and Xiao L (2019) Differential

Expression of Three Cryptosporidium

Species-Specific MEDLE Proteins.

Front. Microbiol. 10:1177.

doi: 10.3389/fmicb.2019.01177

\section{Differential Expression of Three Cryptosporidium Species-Specific MEDLE Proteins}

\author{
Jiayuan $\mathrm{Su}^{1}$, Chanchan Jin ${ }^{1}$, Haizhen $\mathrm{Wu}^{2}$, Jilan $\mathrm{Fei}^{1}$, $\mathrm{Na} \mathrm{Li}^{3}$, Yaqiong Guo ${ }^{3}$, Yaoyu Feng ${ }^{1,3 *}$ \\ and Lihua Xiao ${ }^{3 *}$ \\ 'State Key Laboratory of Bioreactor Engineering, School of Resources and Environmental Engineering, East China University \\ of Science and Technology, Shanghai, China, ' 2 School of Biotechnology, East China University of Science and Technology, \\ Shanghai, China, ${ }^{3}$ Key Laboratory of Zoonosis of Ministry of Agriculture, College of Veterinary Medicine, South China \\ Agricultural University, Guangzhou, China
}

Cryptosporidium parvum and Cryptosporidium hominis share highly similar proteomes, with merely $\sim 3 \%$ divergence in overall nucleotide sequences. Cryptosporidium-specific MEDLE family is one of the major differences in gene content between the two species. Comparative genomic analysis indicated that MEDLE family may contribute to differences in host range among Cryptosporidium spp. Previous studies have suggested that CPMEDLE-1 encoded by cgd5_4580 and CPMEDLE-2 encoded by cgd5_4590 are potentially involved in the invasion of C. parvum. In this study, we expressed in Escherichia coli, the $C$. hominis-specific member of the MEDLE protein family, ChMEDLE-1 encoded by chro.50507, and two C. parvum-specific members, CpMEDLE-3 encoded by cgd5_4600 and CPMEDLE-5 encoded by cgd6_5480. Quantitative PCR, immunofluorescence staining and in vitro neutralization assay were conducted to assess their biologic characteristics. The expression of the cgd5_4600 gene was high during 12-48 h of the in vitro culture, while the expression of cgd6_5480 was the highest at $2 \mathrm{~h}$. ChMEDLE-1 and CPMEDLE-3 proteins were mostly located in the anterior and mid-anterior region of sporozoites and merozoites, whereas CPMEDLE- 5 was expressed over the entire surface of these invasive stages. Polyclonal antibodies against MEDLE proteins had different neutralization efficiency, reaching approximately 50\% for ChMEDLE-1 and $60 \%$ for CpMEDLE-3, but only 20\% for CpMEDLE-5. The differences in protein and gene expression and neutralizing capacity indicated the MEDLE proteins may have different roles during Cryptosporidium invasion and growth.

Keywords: Cryptosporidium parvum, Cryptosporidium hominis, MEDLE family, invasion, growth

\section{INTRODUCTION}

Cryptosporidium spp. are apicomplexan pathogens inhabiting the brush border of the gastrointestinal epithelium of various vertebrates, causing enterocolitis, vomiting, and watery diarrhea (Checkley et al., 2015). Hundreds of waterborne outbreaks of cryptosporidiosis have been reported around the world (Efstratiou et al., 2017). In most immune-competent individuals, the diarrhea lasts 1-2 weeks after the infection. However, immunocompromised persons such as AIDS patients may experience prolonged, life-threatening diarrhea (Chalmers and Davies, 2010). 
There are no effective anti-parasitic drugs for cryptosporidiosis. One of the reasons may be the lack of knowledge of the invasion process of Cryptosporidium spp. (Singh et al., 2015; Bhalchandra et al., 2018).

The genus Cryptosporidium consists of about 100 named species and genotypes that differ from each other in host specificity (Feng et al., 2018). Most of them have a narrow host range; thus, C. hominis mostly infects humans. A few species such as C. parvum have a broader range of hosts, including ruminants, rodents, and humans (Xiao, 2010; Ryan and Hijjawi, 2015). Comparative genomic analysis has shown highly similar proteomes between these two major humanpathogenic species, with merely $\sim 3 \%$ divergence in overall nucleotide sequences (Abrahamsen et al., 2004). Differences in gene content between the two Cryptosporidium species are centered on two major secreted protein families, MEDLE and insulinase-like proteases (Guo et al., 2015). For the MEDLE family, named after its conserved sequence motif at the C-terminus, six subtelomeric genes are present in C. parvum, compared with one in $C$. hominis. It was thus suggested that MEDLE family of secreted proteins could contribute to differences in host specificity between C. parvum and C. hominis (Liu et al., 2016; Feng et al., 2017).

In previous studies, $C$. parvum-specific MEDLE-1 encoded by cgd5_4580 and MEDLE-2 encoded by cgd5_4590 were characterized by our group (Li et al., 2017; Fei et al., 2018). These studies supported the potential involvement of MEDLE proteins in the invasion of C. parvum. While MEDLE proteins share similar amino acid sequence, MEDLE-1 and MEDLE-2 are unusual members in the protein family, as their amino acid sequences end without the signal motif MEDLE at the C-terminus (Guo et al., 2015).

To further explore the functions of different MEDLE proteins, we have expressed in this study recombinant proteins of $C$. hominis-specific MEDLE-1 encoded by the chro.50507 gene, its homolog in C. parvum, CpMEDLE-3 encoded by cgd5_4600, and the C. parvum-specific CpMEDLE-5 encoded by cgd6_5480. Through immunofluorescence microscopy, quantitative analysis of the MEDLE gene expression, and in vitro neutralization assays, we examined the expression profiles and potential roles of the three MEDLE proteins in host cell invasion and parasite growth.

\section{MATERIALS AND METHODS}

\section{Cryptosporidium parvum Oocyst, Host Cells, and Infection Model}

Cryptosporidium parvum oocysts (IOWA strain) were purchased from Waterborne, Inc. (New Orleans, LA, USA), stored in

\footnotetext{
Abbreviations: DAPI, 4',6-Diamidino-2-phenylindole; DIC, Differential interference contrast; ELISA, Enzyme-linked immunosorbent assay; HRP, Horseradish peroxidase; IPTG, Isopropyl b-D-1-thiogalactopyranoside; MALDI-TOF-MS, Matrix-assisted laser desorption/ionization time of flight mass spectrometry; PBS, Phosphatebuffered saline; PcAb, Polyclonal antibodies; SDS-PAGE, Sodium dodecyl sulfate polyacrylamide gel electrophoresis.
}

antibiotics (200 U/ml penicillin, $200 \mu \mathrm{g} / \mathrm{ml}$ streptomycin, and $0.5 \mu \mathrm{g} / \mathrm{ml}$ amphotericin B) at $4^{\circ} \mathrm{C}$, and used within 3 months after their harvest. Cryptosporidium hominis oocysts were purified from feces of naturally infected crab-eating macaques using the sucrose density gradient centrifugation method (Arrowood and Donaldson, 1996) and used within 3 weeks. The identification of Cryptosporidium species for isolates used in this study was made by PCR and sequence analyses of the small subunit rRNA gene (Feng et al., 2014). Prior to infection, C. parvum and $C$. hominis oocysts were treated on ice for $10 \mathrm{~min}$ with $0.5 \%$ sodium hypochlorite and washed three times with PBS. To obtain free sporozoites, sodium hypochlorite-treated oocysts were excysted in sterile PBS supplemented with $0.75 \%$ sodium taurocholate and $0.25 \%$ trypsin at $37^{\circ} \mathrm{C}$ for $1 \mathrm{~h}$. Human ileocecal adenocarcinoma HCT-8 cells were purchased from the Chinese Academy of Sciences Shanghai Branch. Before infection experiments, HCT- 8 cells were seeded into 12 -well cell culture plates and cultured at $37^{\circ} \mathrm{C}$ in a humidified incubator containing $5 \% \mathrm{CO}_{2}$ until they reached $\sim 80 \%$ confluence. Each well was inoculated with sporozoites from $5 \times 10^{5}$ oocysts in RPMI 1640 media containing $10 \%$ fetal bovine serum, 15 mM HEPES, $50 \mathrm{mM}$ glucose, $10 \mu \mathrm{g} / \mathrm{ml}$ of bovine insulin, $35 \mu \mathrm{g} / \mathrm{ml}$ of ascorbic acid, $1.0 \mu \mathrm{g} / \mathrm{ml}$ of folic acid, $4.0 \mu \mathrm{g} / \mathrm{ml}$ of 4 -aminobenzoic acid, $2.0 \mu \mathrm{g} / \mathrm{ml}$ of calcium pantothenate, $50 \mathrm{U} / \mathrm{ml}$ of penicillin $\mathrm{G}$, $50 \mathrm{U} / \mathrm{ml}$ of streptomycin, and $0.25 \mu \mathrm{g} / \mathrm{ml}$ of amphotericin B (Mauzy et al., 2012). After incubation at $37^{\circ} \mathrm{C}$ for $2 \mathrm{~h}$, unexcysted oocysts and free sporozoites were washed off the monolayers with sterile PBS. Fresh RPMI 1640 medium containing 2\% fetal bovine serum was added to allow cells to grow for specified periods.

\section{Cloning of MEDLE Genes and Construction of Recombinant Plasmids}

As MEDLE genes have no introns, we used Cryptosporidium genomic DNA to amplify the coding sequences of the target genes. The template DNA was extracted from Cryptosporidium oocysts by using the Qiagen DNeasy Blood \& Tissue Kit (Qiagen, Hilden, Germany). Using primers incorporated with restriction sites, the cgd5_4600 gene (XM_625308) and cgd6_5480 (XM_625312) genes were amplified by PCR from C. parvum DNA while the chro.50507 gene (XM_660938), the homolog of $c g d 5 \_4600$, from C. hominis DNA. The primers used included 5' -CCGGAATTCTTTTTGGTTAAAAAAGATG-3' (including an EcoRI restriction site) and 5'-CCGCTCGAGATA TTTTTCCATGACCCAC-3' (including an XhoI restriction site) for chro.50507, 5' -AAATCCATGGAAAATATAACCGATAATT-3' (including an NcoI restriction site) and 5'-AAAT $\underline{C}$ TCGAGTTCCAAATCTTCCATATTAA-3' (including an XhoI restriction site) for cgd5_4600, and 5'-CGGAATTC ACCGATGATTTTTTGGTTAA3' (including an EcoRI restriction site) and 5' -AAATCTCGAGTTCCAAATCTTCCATATTAATA-3' (including an XhoI restriction site) for cgd6_5480. The 50- $\mu 1$ PCR reaction contained $1 \mu \mathrm{l}$ template DNA, $0.25 \mathrm{mM}$ primers, $3 \mathrm{mM} \mathrm{MgCl}_{2}, 200 \mu \mathrm{M}$ deoxynucleotide triphosphates, $1 \times$ GeneAmp PCR buffer (Applied Biosystems, Foster City, $\mathrm{CA}$ ), and $1.5 \mathrm{U}$ Taq polymerase (Promega, Madison, WI). 
The amplification was conducted on a GeneAmp 9700 (Applied Biosystems) using an initial denaturation at $94^{\circ} \mathrm{C}$ for $5 \mathrm{~min}$; 35 cycles of amplification at $94^{\circ} \mathrm{C}$ for $45 \mathrm{~s}, 52^{\circ} \mathrm{C}$ for $45 \mathrm{~s}$, and $72^{\circ} \mathrm{C}$ for $1 \mathrm{~min}$; and one final extension at $72^{\circ} \mathrm{C}$ for $7 \mathrm{~min}$. The PCR products were purified by using the Column PCR Product Purification Kit (Qiagen), digested with EcoRI/ $\mathrm{NcoI}$ and XhoI, and ligated into double-digested vector pET28a (Novagen, Madison, WI). The recombinant plasmids were transformed into competent E. coli $\mathrm{DH} 5 \alpha$ (Tiangen, Beijing, China). Positive colonies selected from solid Luria-Bertani (LB) ager containing $50 \mu \mathrm{g} / \mathrm{ml}$ of kanamycin were identified by PCR. They were sequenced to verify their identity and sequence accuracy.

\section{Expression of Recombinant MEDLE Protein in E. coli}

To express the target proteins, recombinant plasmids were extracted from E. coli $D H 5 \alpha$ using the QIAGEN Plasmid Mini Kit (Qiagen) and transformed into E. coli BL21 (DE3) (Tiangen), which was incubated at $37^{\circ} \mathrm{C}$ in liquid $\mathrm{LB}$ medium supplemented with $50 \mu \mathrm{g} / \mathrm{ml}$ of kanamycin. When the $\mathrm{OD}_{600}$ reached $0.6,0.5 \mathrm{mM}$ isopropyl b-D-1-thiogalactopyranoside (IPTG) was introduced to induce protein expression at $25^{\circ} \mathrm{C}$ for $8 \mathrm{~h}$. The expression level of target proteins was assessed by using sodium dodecyl sulfate polyacrylamide gel electrophoresis (SDS-PAGE) and western blot analyses. The loading buffer for the SDS-PAGE contained dl-dithiothreitol (DTT) and sodium dodecyl sulfate (SDS).

\section{Purification of MEDLE Proteins and Preparation of Anti-MEDLE Antibodies}

The target proteins were purified using Ni-NTA beads (Novagen) and manufacturer-recommended procedures. In brief, cultured $E$. coli cells were harvested by centrifugation and lysed by sonication. The soluble fraction of the E. coli lysate was collected by centrifugation at $10,000 \mathrm{~g}$ for $30 \mathrm{~min}$. After filtered through a $0.45 \mu \mathrm{m}$ cellulose acetate membrane filter (Millipore, Billerica, MA), the supernatant was loaded onto Ni-NTA beads. After washing with five volumes of $20 \mathrm{mM}$ imidazole buffer, target proteins were eluted with buffers containing increasing concentrations of imidazole. The purity and quantity of recombinant proteins harvested were assessed by using SDS-PAGE and a BCA kit (Yeasen, Shanghai, China). The identity of the target proteins was confirmed by using Matrix-Assisted Laser Desorption/ Ionization Time of Flight Mass Spectrometry (MALDITOF-MS) (Murugaiyan and Roesler, 2017).

Polyclonal antibodies to all MEDLE proteins examined in this study were produced through immunizations of rabbits by GL Biochem Ltd (Shanghai, China). In the primary immunization of healthy New Zealand white rabbits, $350 \mu \mathrm{g}$ of the purified protein mixed with Freund's complete adjuvant was used. Boosted immunizations were conducted every 7 days for six times using $150 \mu \mathrm{g}$ purified protein in the Freund's incomplete adjuvant. Seven days after the last immunization, post-immune sera were harvested from the immunized animals.
Pre-immune sera prior to the primary immunization were collected as negative controls. Polyclonal IgG antibodies were purified from the immune sera using protein A sepharose affinity chromatography.

\section{Assessment of Cross-Reactivity of Anti-MEDLE Antibodies}

To assess the cross-reactivity of anti-MEDLE antibodies, the three recombinant MEDLE proteins produced in this study as well as CPMEDLE-1 and CPMEDLE-2 were employed as antigens in enzyme-linked immunosorbent assays (ELISA). They were coated onto ELISA plates at the concentration of $2 \mu \mathrm{g} / \mathrm{ml}$. Antibodies against CpMEDLE-3, CpMEDLE-5 and ChMEDLE- 1 and horseradish peroxidase (HRP)-conjugated goat-anti-rabbit secondary antibodies (Yeasen) were incubated in turn for the detection of reactions to the coated antigens by individual antibodies, with the pre-immune sera being used as the negative control. The reactivity of individual antibodies to each MEDLE protein was evaluated by absorbance at $450 \mathrm{~nm}$. The optical density of negative control was used to compute the relative absorbance of each reaction. For example, the relative absorbance of anti-CpMEDLE-3 antibodies to CPMEDLE-5 antigen was calculated by the equation $V_{C p 3-C p 5}=\frac{O D_{C p 3-C p 5}}{O D_{N-C p 5}}$, where $O D_{C p 3-C p 5}$ is the difference in optical density of the reaction between anti-CpMEDLE-3 and CpMEDLE-5, and $O D_{N-C p 5}$ is the difference in optical density of the reaction between pre-immune sera and CPMEDLE-5.

The relative absorbance of each antibody to corresponding protein was used to normalize the final data. The reactive cross-reactivity was calculated using the following formula:

Relative cross-reactivity of antibodies to antigen A against antigen $\mathrm{B}=\frac{V_{A-B}}{V_{A-A}}=\frac{O D_{A-B} / O D_{N-B}}{O D_{A-A} / O D_{N-A}}$.

Western blot analysis was conducted to further assess the cross-reactivity of anti-MEDLE antibodies as described above.

\section{Localization of MEDLE Protein Expression on Sporozoites and Developmental Stages}

The reactivity of anti-MEDLE antibodies to native proteins (in crude protein extract from $1 \times 10^{7}$ oocysts/lane) in C. parvum sporozoites was assessed by using western blot as described (Li et al., 2017). Polyclonal antibodies against MEDLE proteins were used as primary antibodies at the 1:4,000 dilution in PBS, while HRP-conjugated goat anti-rabbit IgG (Yeasen) as secondary antibodies at the 1:5,000 dilution in PBS, with pre-immune sera as controls. To examine MEDLE protein expression in life cycle stages, free sporozoites and intracellular parasites in HCT- 8 cells on slides harvested 24 and $48 \mathrm{~h}$ after infection were fixed with $4 \%$ paraformaldehyde in PBS for $30 \mathrm{~min}$. The fixed cells were permeabilized with $0.5 \%$ Triton X-100 in PBS for $15 \mathrm{~min}$. After three washes with sterile PBS, the slides were incubated with DB Blocking Buffer (B100-40, Waterborne Inc.) at room 
temperature for $30 \mathrm{~min}$. After another three washes with PBS, the slides were incubated with polyclonal antibodies against individual MEDLE proteins (1:8,000 diluted in PBS) for $1 \mathrm{~h}$. Alexa Fluor $^{\circledR}$ 594-conjugated Goat Anti-rabbit IgG (Cell Signaling Technology, Danvers, MA) diluted 1:400 was used as the secondary antibodies in the immunofluorescence assay (Jakobi and Petry, 2006). Nuclei of organisms were counterstained by using 4',6-diamidino2-phenylindole (DAPI, Roche, Basel, Switzerland). The slides were finally mounted with No-Fade Mounting Medium (Boster, Wuhan, China), with coverslips being sealed with nail enamel. They were examined using a BX53 immunofluorescence microscope (Olympus, Tokyo, Japan) under the $400 \times$.

\section{Quantitative Analysis of MEDLE Gene Expression by qPCR}

The expression levels of MEDLE genes during in vitro development of $C$. parvum were evaluated by using qPCR. Similar work was not conducted on $C$. hominis because of the unavailability of large numbers of fresh oocysts. Total RNA was isolated from C. parvum-infected HCT-8 cells at 0, 2, 6, $12,24,36,48$, and $72 \mathrm{~h}$ by using the RNeasy Plus Mini Kit (Qiagen). Afterward, cDNA was synthesized from $1 \mu \mathrm{g}$ of the total RNA using the RevertAid First Strand cDNA Synthesis Kit (Thermo Fisher Scientific). The expression of individual MEDLE genes was assessed by using qPCR analysis of the cDNA, with the final expression data being normalized with data on the expression of the C. parvum 18S rRNA (Cp18S rRNA) gene (Cai et al., 2005). Each 20- $\mu$ l qPCR reaction consisted of $1 \times$ SYBR Green Supermix (TOYOBO, Osaka, Japan), $0.1 \mathrm{mM}$ primers, and $1 \mu \mathrm{l} \mathrm{cDNA}$. The primers used for qPCR were as follows: primers for the CP18S rRNA: 5'-CTAGAGATTGGAGGTTGTTCC-3' and 5' -CTCCACCAAC TAAGAACGGC -3' (amplicon size $=256$ bp) (Mauzy et al., 2012); primers for the cgd5_4600 gene: $5^{\prime}$-ATGATCCG TTCGTCGCTTAC-3' and 5' -ACGCCGATGTTTTCTACCTG-3' (amplicon size $=243 \mathrm{bp}$ ); and primers for the cgd6_5480 gene:


ATGGTTGT-3' (amplicon size $=146$ bp). The $\mathrm{qPCR}$ amplifications were performed on a Light Cycler 480 (Roche) for 45 cycles $\left(95^{\circ} \mathrm{C}\right.$ for $30 \mathrm{~s}, 58^{\circ} \mathrm{C}$ for $30 \mathrm{~s}$, and $72^{\circ} \mathrm{C}$ for $30 \mathrm{~s}$ ), with an initial denaturation at $95^{\circ} \mathrm{C}$ for $1 \mathrm{~min}$. The amplification was followed by a melt curve analysis through $95^{\circ} \mathrm{C}$ for $1 \mathrm{~min}$, ramping down to $57^{\circ} \mathrm{C}$ in $2^{\circ}$ intervals, and ramping back to $95^{\circ} \mathrm{C}$ for $30 \mathrm{~s}$. The threshold cycle $\left(C_{\mathrm{T}}\right)$ values from the qPCR were used in computing the relative levels of gene expression using the $\Delta \Delta C_{T}$ approach (Livak and Schmittgen, 2001). Three biological replicates and two technical replicates were used in each experiment. The mean values in this report were obtained from three independent experiments.

\section{In vitro Neutralization of Sporozoite Invasion}

The potential involvement of MEDLE proteins in host cell invasion by C. hominis and C. parvum sporozoites was examined using neutralization assays (Kuhlenschmidt et al., 2016). HCT-8 cells were infected with sporozoites of $C$. hominis $\left(1 \times 10^{5}\right.$ oocysts/well $)$ or C. parvum $\left(5 \times 10^{5}\right.$ oocysts/well $)$ in the presence of immune sera, anti-MEDLE polyclonal antibodies, or pre-immune sera. The sera were used at 1:200, 1:500, and 1:1,000 dilutions, while anti-MEDLE polyclonal antibodies were used at 1:4,000 dilutions. After 2-h incubation, free sporozoites were washed off the culture, which was allowed to continue for additional $24 \mathrm{~h}$. Cy3-labeled polyclonal antibodies against C. parvum sporozoites (Waterborne, Inc.) were used to label developmental stages as described (Upton et al., 1995). The stained culture was examined under an immunofluorescence microscope as described above. Images were taken under the $200 \times$ and analyzed using the ImageJ software ${ }^{1}$. The mean parasite load per field was obtained from 50 random fields from each culture. Data from two (for C. hominis due to the limited availability of fresh oocysts) or three (for C. parvum) independent experiments were compared among treatment groups using the Student's $t$ test. Data from cultures treated with pre-immune sera were used to normalize neutralization effects of immune sera.

\section{RESULTS}

\section{Production of Recombinant MEDLE Proteins in E. coli}

We successfully cloned the three MEDLE genes into the pET28a vector (Figure 1A). The recombinant plasmids were sequenced to verify the identity and accuracy of DNA sequences. All three recombinant MEDLE proteins were expressed successfully at the predicted size of $\sim 20 \mathrm{kDa}$ (Figure 1B), although in western blot analysis another band of $\sim 40 \mathrm{kDa}$ was seen in products from all three recombinant plasmids (Figure 1C). Results of MALDI-TOF-MS analysis confirmed their identity, with the larger band possibly representing the dimers of the MEDLE proteins. Pure recombinant MEDLE proteins were obtained using the Ni-NTA purification method through the His-tag incorporated (Figure 1D).

\section{Cross-Reactivity of Antibodies to MEDLE Family Proteins}

In western blot analysis, anti-CpMEDLE-3 antibodies showed significant cross-reactivity to ChMEDLE-1, and mainly reacted with the $\sim 40 \mathrm{kDa}$ dimers of CPMEDLE-3 and ChMEDLE-1 (Figure 2A). Anti-ChMEDLE-1 antibodies reacted with $\sim 20 \mathrm{kDa}$ monomers of ChMEDLE-1 and the $\sim 40 \mathrm{kDa}$ dimers of CPMEDLE-3, with weak reactivity to other MEDLE proteins. In contrast, anti-CpMEDLE-5 antibodies showed minimum cross-reactivity to other MEDLE proteins. Similar observations were made in ELISA analysis of the cross-reactivity, with CPMEDLE- 3 and ChMEDLE- 1 being recognized by ChMEDLE-1 and CPMEDLE-3 antibodies, respectively, and minimum reactivity of CpMEDLE- 1 or CPMEDLE-5 antibodies to other MEDLE proteins (Figure 2B). Thus, anti-ChMEDLE-1 antibodies were expected to react with $C$. parvum sporozoites due to its cross-reactivity to CPMEDLE-3.

${ }^{1}$ https://imagej.nih.gov/ij/ 


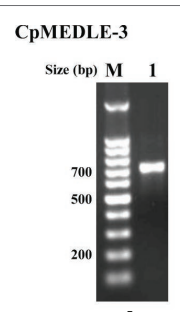

A

CpMEDLE-5

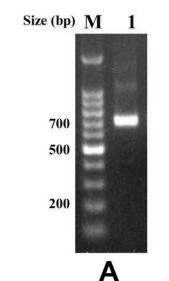

A

\section{ChMEDLE-1}

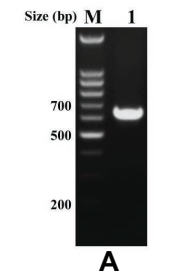

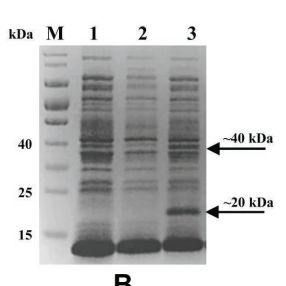

B

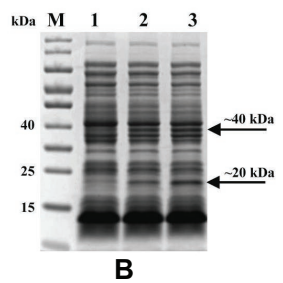

B

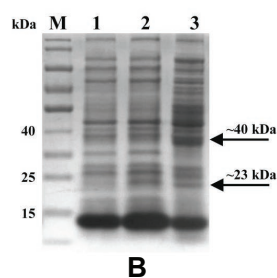

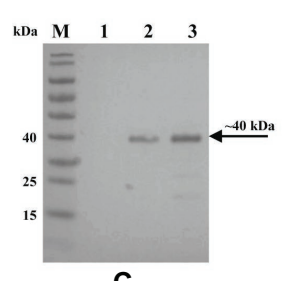

C



C

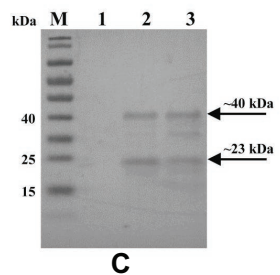

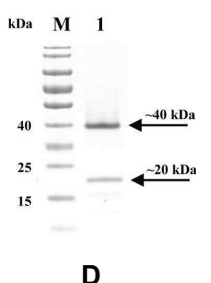

D
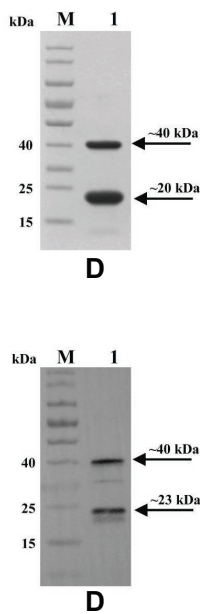

FIGURE 1 | Production and purification of recombinant MEDLE proteins CpMEDLE-3 and CpMEDLE-5 from Cryptosporidium parvum and ChMEDLE-1 from Cryptosporidium hominis. (A) PCR amplification of the target gene in genomic DNA. Lane M: molecular markers; lane 1: PCR product. (B) Expression of recombinant MEDLE protein in E. coli BL21 (DE3) as revealed by SDS-PAGE analysis. Lane M: molecular weight markers; lane 1: lysate from bacteria culture transformed with the recombinant plasmid without IPTG induction; lane 2: Iysate from similar bacteria culture induced by IPTG for 2 h; lane 3: Iysate from bacteria culture induced by IPTG for $8 \mathrm{~h}$, with the expected product indicated by an arrow. (C) Western blot analysis of the recombinant protein. Lane M: molecular weight markers; lane 1: Iysate from bacteria culture transformed with recombinant plasmid without IPTG induction; lane 2: supernatant from IPTG-induced bacterial culture; lane 3: cell lysate from IPTG-induced bacterial culture. (D) Purification of recombinant proteins. Lane M: molecular weight markers; lane 1: purified recombinant proteins from Ni-NTA affinity chromatography.

\section{Expression of MEDLE Proteins in C. parvum Sporozoites}

All three anti-MEDLE antibodies or post-immune sera recognized their corresponding MEDLE proteins, while pre-immune sera did not react with sporozoites proteins and recombinant proteins in western blot assay (Figures $\mathbf{3 A}, \mathbf{4 A}, \mathbf{5 A}$ ). The monomers and dimers of the three recombinant MEDLE proteins were all detected by using anti-MEDLE antibodies or post-immune sera. Native MEDLE proteins in C. parvum sporozoite extracts were recognized by the anti-CPMEDLE- 3 antibodies and immune sera, generating two bands of $\sim 15$ and $\sim 40 \mathrm{kDa}$ (Figure 3A). A band of $\sim 15 \mathrm{kDa}$ protein in sporozoite extracts was recognized by CPMEDLE- 5 antibodies and immune sera, but no dimerization of CpMEDLE-5 was observed (Figure 4A). Western blot analysis of native proteins was not conducted for C. hominis due to limited oocyst availability.

\section{Localization of MEDLE Proteins on Sporozoites and Developmental Stages}

The expression location of MEDLE proteins in sporozoites and developmental stages was examined by immunofluorescence microscopy. CPMEDLE-3 antibodies reacted mostly with the anterior and mid-anterior regions of C. parvum sporozoites (Figure 3B), while CPMEDLE-5 antibodies reacted with the entire surface of $C$. parvum sporozoites (Figure 4B). The expression of CPMEDLE-3 on C. parvum sporozoites was also confirmed by using anti-ChMEDLE-1 antibodies (Figure 5B). ChMEDLE-1 was expressed mainly in the mid-anterior region of C. hominis sporozoites (Figure 5B). No reactivity to C. parvum or C. hominis sporozoites was detected using pre-immune sera (data not shown).

In assessment of MEDLE expression in intracellular stages at 24 and $48 \mathrm{~h}$ of cultures, CPMEDLE- 3 and ChMEDLE- 1 antibodies reacted with only part of the merozoites opposite to the nucleus (Figures 3B, 5B). In contrast, the reactivity of CPMEDLE-5 antibodies dispersed throughout the meronts, including the merozoites within them (Figure 4B). Antibodies against all three MEDLE proteins did not recognize the parasitophorous vacuole.

\section{Expression of MEDLE Gene in C. parvum Culture}

The relative expression levels of the MEDLE genes during intracellular development of $C$. parvum in cell culture during 


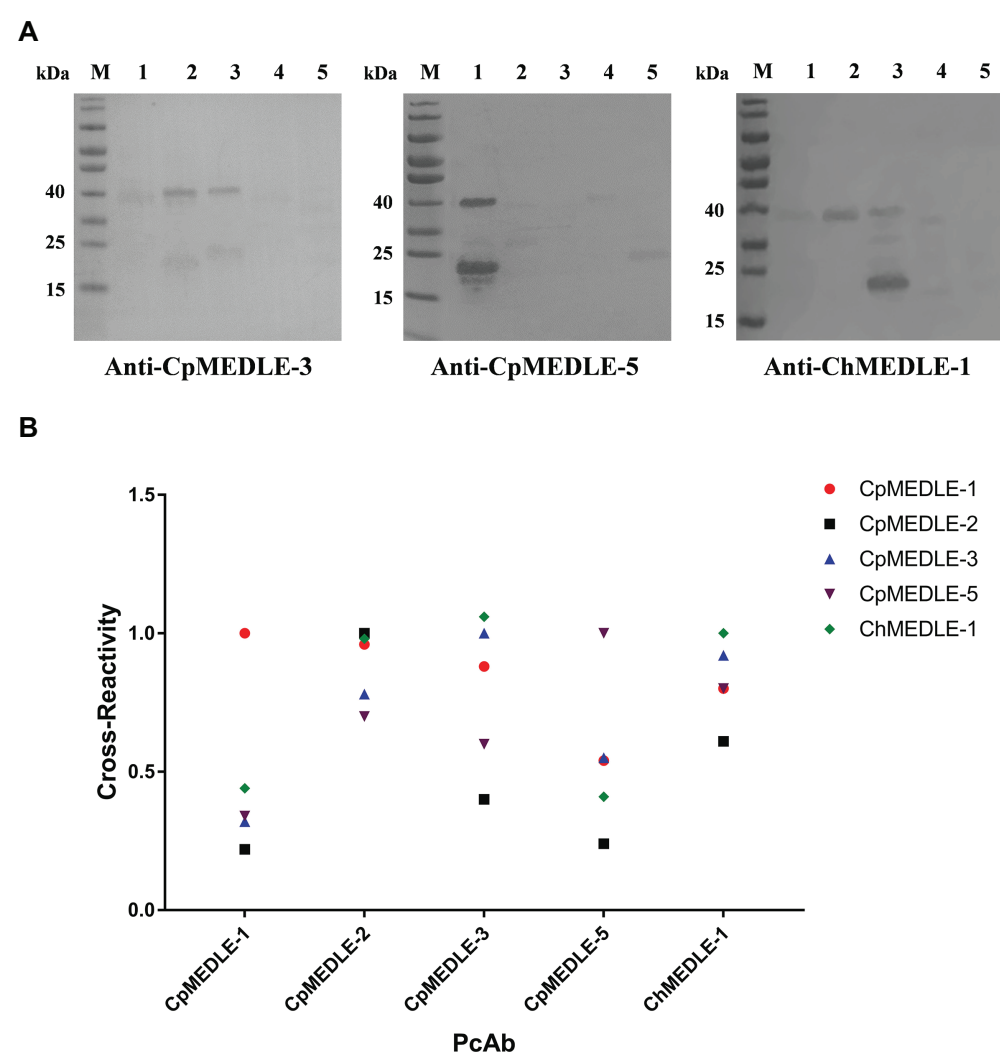

FIGURE 2 | Cross-reactivity of polyclonal antibodies (PCAb) among MEDLE proteins. (A) Western blot analysis of cross-reactivity among MEDLE proteins, using $1 \mu \mathrm{g}$ of CpMEDLE-5 (lane 1), CpMEDLE-3 (lane 2), ChMEDLE-1 (lane 3), CpMEDLE-1 (lane 4) and CpMEDLE-2 (lane 5) and PcAb to CpMEDLE-3 (left panel), CPMEDLE-5 (middle panel) and ChMEDLE-1 (right panel). (B) ELISA analysis of polyclonal antibodies to each MEDLE protein, with data being normalized using the relative absorbance of each antibody to the corresponding protein.

the first $72 \mathrm{~h}$ were assessed by qPCR in three independent experiments. The expression of the $c g d 5 \_4600$ gene increased gradually during the parasite development, with high levels of expression sustained from 24 to $48 \mathrm{~h}$ of culture (Figure 6A, left panel). In contrast, the expression of the cgd6_5480 gene was highest at $2 \mathrm{~h}$ of culture, with a drastic reduction thereafter till $72 \mathrm{~h}$ (Figure 6B, left panel). The expression of the chro.50507 gene for ChMEDLE-1 in C. hominis was not assessed due to the lack of sufficient fresh oocysts.

\section{Neutralization of Sporozoite Invasion by MEDLE Antibodies}

Invasion neutralization assays were used to assess the effect of anti-MEDLE antibodies on the infection of $\mathrm{HCH}-8$ cells with C. parvum (in three independent experiments) or C. hominis (in two independent experiments). Compared with control cultures, a significant reduction in C. parvum load was seen when sporozoites were pre-incubated with antisera from CpMEDLE-3 immunized rabbits (Figure 6A, right panel). The inhibition rate was $30.1 \%$ [39.5 \pm 5.6 and $27.6 \pm 4.0$ per $200 \times$ field for pre- and post-immune sera, respectively; $t_{(2)}=12.931, p=0.006$ ] at $1: 1,000$ dilution, 53.9\% [38.8 \pm 5.4 and $17.9 \pm 2.6$ per $200 \times$ field for pre- and post-immune sera, respectively; $t_{(2)}=12.144, p=0.007$ ] at 1:500 dilution, and $56.5 \%$ [40.7 \pm 5.0 and $17.7 \pm 1.8$ per $200 \times$ field for pre- and post-immune sera, respectively; $t_{(2)}=8.549, p=0.013$ ] at 1:200 dilution. The parasite load in cultures with no addition of rabbit sera was $39.1 \pm 4.8$ per $200 \times$ field. In contrast, antisera generated from CPMEDLE-5 immunization did not have any similar effects on C. parvum invasion (Figure 6B, right panel), with only $10.3 \%[64.7 \pm 2.4$ and $58.1 \pm 2.4$ per $200 \times$ field for pre- and post-immune sera, respectively; $\left.t_{(2)}=7.551, p=0.017\right], 12.8 \%[61.5 \pm 4.9$ and $53.7 \pm 2.1$ per $200 \times$ field for pre- and post-immune sera, respectively; $\left.t_{(2)}=4.594, p=0.044\right]$, and $22.6 \%[61.5 \pm 4.0$ and $47.6 \pm 2.2$ per $200 \times$ field for pre- and post-immune sera, respectively; $\left.t_{(2)}=4.329, p=0.049\right]$ reductions at 1:1,000, 1:500, and 1:200 dilutions, respectively.

In neutralization assays conducted with antisera from ChMEDLE-1 immunization, when HCT-8 cells were infected with C. hominis (Figure 6C, left panel), the highest inhibition rate reached $40.6 \%$ at $1: 100$ dilution of immune sera [14.2 \pm 0.8 and $8.5 \pm 0.2$ per $200 \times$ field for pre- and post-immune sera, respectively; $\left.t_{(1)}=14.073, p=0.045\right]$. A similar inhibition of C. hominis invasion was achieved with the addition of purified anti-ChMEDLE-1 antibodies at 1:4,000 dilution: 43.1\% [14.5 \pm 1.0 and $8.1 \pm 0.4$ per $200 \times$ field for no addition culture and polyclonal antibodies, respectively; $\left.t_{(1)}=15.190, p=0.042\right]$. 

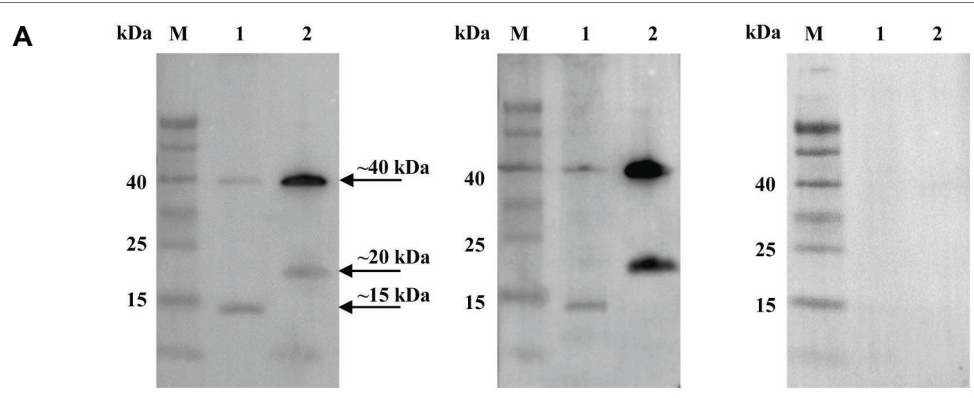

B


FIGURE 3 | Expression of CPMEDLE-3 in sporozoites and developmental stages of Cryptosporidium parvum. (A) Western blots analysis of native protein from C. parvum sporozoites for CpMEDLE-3, using polyclonal antibodies (left panel), post-immune sera (middle panel) and pre-immune sera (right panel). Lane M: molecular weight markers; lane 1: crude protein extracted from sporozoites; lane 2: purified CpMEDLE-3 protein. (B) Expression of CpMEDLE-3 on C. parvum sporozoites (top panel) and intracellular developmental stages in HCT-8 cell cultures at $24 \mathrm{~h}$ (middle panel) and $48 \mathrm{~h}$ (bottom panel). The images were taken under differential interference contrast (DIC), with nucleus counter-stained with 4',6-diamidino-2-phenylindole (DAPI), parasites stained by immunofluorescence with Alexa 594-labled CPMEDLE-3 (CPMEDLE-3), and superimposition of the three images (Merged). Bars $=5 \mu \mathrm{m}$.

When HCT-8 cell culture was inoculated with C. parvum sporozoites treated with antisera against ChMEDLE-1 (Figure 6C, right panel), the inhibition rate was $17.3 \%[70.7 \pm 2.3$ and $58.5 \pm 3.7$ per $200 \times$ field for pre- and post-immune sera, respectively; $\left.t_{(2)}=15.408, p=0.004\right], 44.2 \%[69.4 \pm 2.4$ and $38.7 \pm 2.7$ per $200 \times$ field for pre- and post-immune sera, respectively; $\left.t_{(2)}=16.424, p=0.004\right]$, and $47.3 \%[67.8 \pm 1.6$ and $35.7 \pm 4.0$ per $200 \times$ field for pre- and post-immune sera, respectively; $\left.t_{(2)}=14.396, p=0.005\right]$ at $1: 500,1: 200$, and $1: 100$ dilutions, respectively.

\section{DISCUSSION}

In this study, we provided evidence for the hypothesis that members of MEDLEs may have different expression and roles in the invasion and growth of C. parvum and C. hominis, although the precise functions of these proteins remain unclear. Most proteins in the Cryptosporidium proteome are encoded by single-copy genes. The MEDLE proteins are among the few Cryptosporidium-specific proteins encoded by gene families. The multi-copy nature of these proteins signals their biological importance. As members of the protein family have similar sequences, they could serve as a strategy for the parasite to diversify its interactions with host cells, especially during the invasion process, such as increased range of susceptible hosts or host cells (reduced host specificity or tissue tropism). Alternatively, these genes have regulated expression, producing different members exerting their functions at specific life cycle stages. Both scenarios appear likely for C. parvum, which has a broader host range C. hominis. The latter, however, has only one copy of the MEDLE genes, but appears to have the invasion process and growth pattern similar to C. parvum.

Despite the significant sequence similarity among MEDLE proteins, they have different spectrum of cross-reactivity of antibodies raised against individual recombinant proteins. Some of the antibodies are more specific, such as those raised against CPMEDLE-1 and CPMEDLE-5, which showed minimum reactivity to other MEDLE proteins. Others, especially those against CPMEDLE-2, cross-react mostly with other MEDLE proteins. ChMEDLE-1 and CPMEDLE-3 have very similar spectrum of antibody cross-reactivity, probably due to their high amino acid sequence identity (67\%). In the native protein expression, CpMEDLE-3 is in the forms of monomer $(\sim 15 \mathrm{kDa})$ and dimer $(\sim 40 \mathrm{kDa})$, while CpMEDLE-5 forms only monomer $(\sim 15 \mathrm{kDa})$. In previous studies, CpMEDLE-2 did not form dimers either, while 

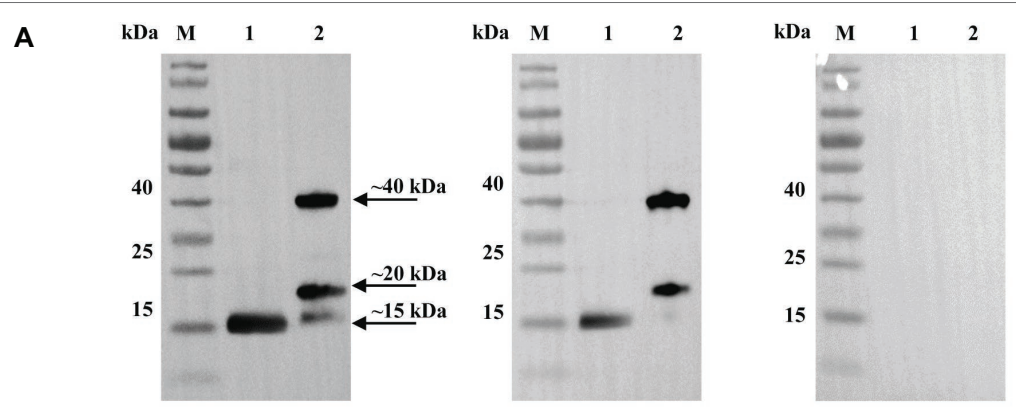

B
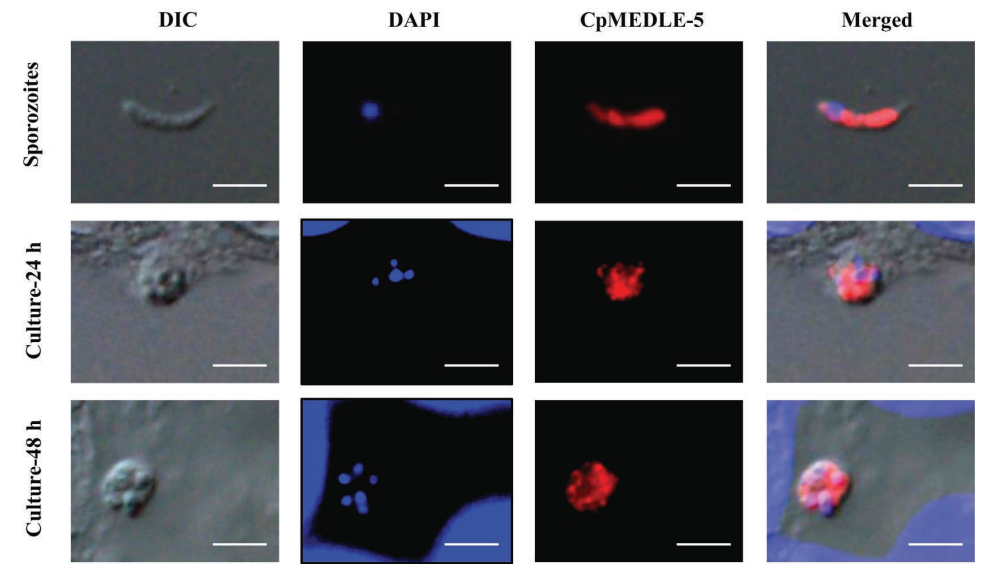

FIGURE 4 | Expression of CPMEDLE-5 in sporozoites and developmental stages of Cryptosporidium parvum. (A) Western blots analysis of native protein from C. parvum sporozoites for CpMEDLE-5, using polyclonal antibodies (left panel), post-immune sera (middle panel) and pre-immune sera (right panel). Lane M: molecular weight markers; lane 1: crude protein extracted from sporozoites; lane 2: purified CpMEDLE-5 protein. (B) Expression of CpMEDLE-5 on C. parvum sporozoites (top panel) and intracellular developmental stages in HCT-8 cell cultures at $24 \mathrm{~h}$ (middle panel) and $48 \mathrm{~h}$ (bottom panel). The images were taken under differential interference contrast (DIC), with nucleus counter-stained with 4',6-diamidino-2-phenylindole (DAPI), parasites stained by immunofluorescence with Alexa 594-labled CpMEDLE-5 (CpMEDLE-5), and superimposition of the three images (Merged). Bars $=5 \mu \mathrm{m}$.

CPMEDLE-1 reacted with fragments of $\sim 32, \sim 36$, and $\sim 50 \mathrm{kDa}$ in C. parvum sporozoite extracts (Li et al., 2017; Fei et al., 2018). These differences in dimer formation among MEDLE proteins in sporozoites support the conclusion on their differential roles. It would be important to determine whether dimerization is critical to the functions or activities of MEDLE proteins.

The functional diversity of MEDLE proteins is supported by their expression patterns in sporozoites and developmental stages. All MEDLE proteins are expressed on both sporozoites and merozoites, the invasive stages of Cryptosporidium spp. CPMEDLE- 3 is expressed in the anterior and mid-anterior regions of C. parvum sporozoites, as indicated by immunofluorescence analyses using both anti-CPMEDLE-3 and anti-ChMEDLE-1 antibodies. In contrast, CPMEDLE-1 and ChMEDLE- 1 are similarly expressed, while CPMEDLE-2 and CPMEDLE-5 are expressed over almost the entire C. parvum sporozoites (Li et al., 2017; Fei et al., 2018). Previous studies reported that most invasion-associated proteins were located in anterior or mid-anterior regions of sporozoites (Singh et al., 2015). Thus, among the three proteins analyzed in the present study, CPMEDLE-3 and ChMEDLE-1 are more likely to play some roles in the invasion of Cryptosporidium. As MEDLE proteins are secretory proteins, these differences in expression patterns could be due to their different subcellular locations. In particular, the dotted location of ChMEDLE-1 on sporozoites and merozoites suggests that it could be a dense granule protein. As the homolog of ChMEDLE-1 in C. parvum, CpMEDLE-3 as expectedly has a similar expression pattern on sporozoites and merozoites; thus, could also be a dense granule protein, while CpMEDLE-2 and CPMEDLE- 5 could be cytoplasmic proteins. Dense granule proteins are known to play critical roles in the invasion and virulence of other apicomplexan parasites such as Toxoplasma gondii (Gubbels and Duraisingh, 2012).

The gene expression profile of MEDLE proteins further illustrates their differential roles in invasion and growth of C. parvum. The genes encoding these proteins have peak expressions at different time during parasite invasion and growth; genes encoding CPMEDLE- 1 and CPMEDLE-5 have the highest expression at $2 \mathrm{~h}$ after invasion, the gene for CpMEDLE-2 peaks at $48 \mathrm{~h}$, while the one for CPMEDLE-3 has high expression after $12 \mathrm{~h}$ ( $\mathrm{Li}$ et al., 2017; Fei et al., 2018). The differences in protein subcellular locations and 

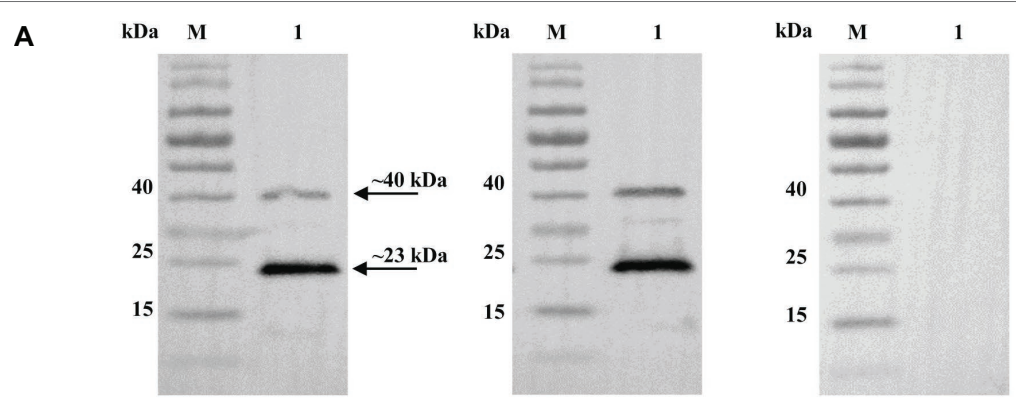

B
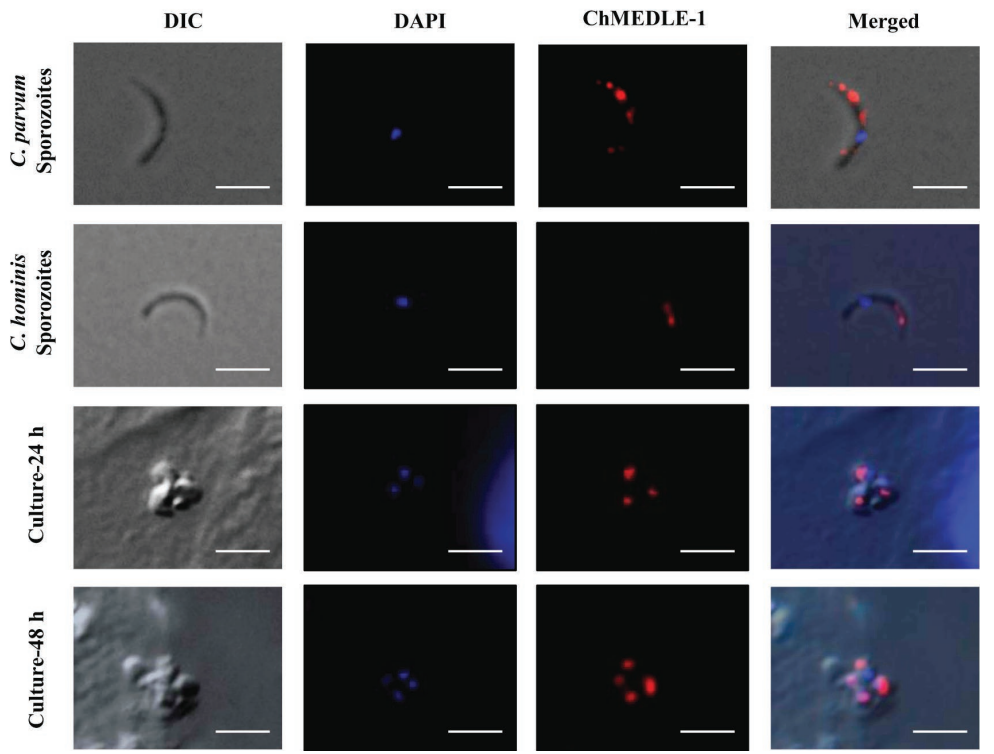

FIGURE 5 | Expression of ChMEDLE-1. (A) Western blots analysis of ChMEDLE-1 recombinant protein, using polyclonal antibodies (left panel), post-immune sera (middle panel) and pre-immune sera (right panel). Lane M: molecular weight markers; lane 1: purified ChMEDLE-1 protein. (B) Expression of ChMEDLE-1 on C. parvum sporozoites (top panel), C. hominis sporozoites (the second panel) and intracellular developmental stages of $C$. hominis in HCT-8 cell cultures at $24 \mathrm{~h}$ (the third panel) and $48 \mathrm{~h}$ (bottom panel). The images were taken under differential interference contrast (DIC), with nucleus counter-stained with 4',6-diamidino-2phenylindole (DAPI), parasites stained by immunofluorescence with Alexa 594-labled ChMEDLE-1 (ChMEDLE-1), and superimposition of the three images (Merged). Bars $=5 \mu \mathrm{m}$

gene transcription patterns agree with the theory that MEDLE proteins play different roles during parasite invasion and growth of C. parvum. The lack of some of the MEDLE proteins in $C$. hominis could be responsible for some of the biological differences between the two Cryptosporidium species, including host specificity.

In neutralization examination, polyclonal antibodies against individual MEDLE proteins have different abilities in inhibiting parasite invasion of HCT-8 cells. For instance, CpMEDLE-5 antibodies inhibited the invasion efficiency only marginally, while antibodies against CPMEDLE-3 reduced the invasion by nearly $60 \%$. The inhibition rate obtained in this study is similar to rates reported in previous studies of several invasion-related proteins. For instance, antisera against gp40, a protein which was involved in $C$. parvum-host cell interactions, inhibited C. parvum infection by $68 \%$ (Cevallos et al., 2000). Polyclonal antibodies to EF-1 $\alpha$, which forms an essential component of the invasion apparatus of the parasite, blocked C. parvum infection of HCT- 8 cells by $80.9 \%$ in a neutralization assay (Matsubayashi et al., 2013). Differences in the neutralization efficiency of antibodies could be due to differences in the roles of various MEDLE proteins during invasion and growth. Among the five MEDLE proteins of $C$. parvum and $C$. hominis examined thus far, CPMEDLE-3 and its homolog ChMEDLE-1, both apparently dense granule proteins, have generated the highest inhibition of Cryptosporidium invasion, indicating their important roles in the invasion of $C$. parvum and C. hominis. Interestingly, ChMEDLE- 1 is the only MEDLE member in C. hominis. Antibodies against ChMEDLE-1 showed significant ability to neutralize the infection of HCT-8 by both C. hominis and C. parvum. As ChMEDLE-1 is the homolog of CPMEDLE-3 in $C$. parvum, the neutralization of $C$. parvum infection by of antibodies against ChMEDLE- 1 is expected. As there was minimal cross-reactivity in antibodies among other MEDLE 
A

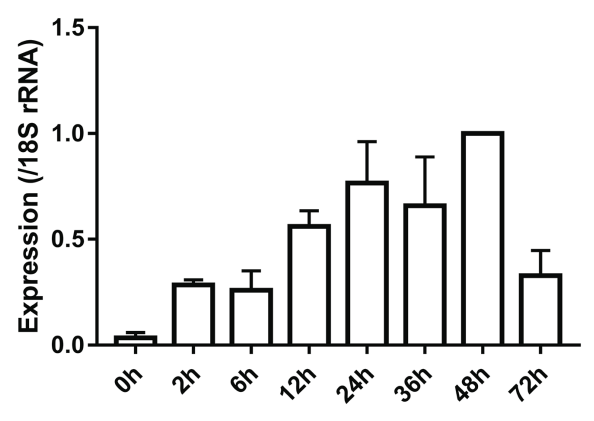

B

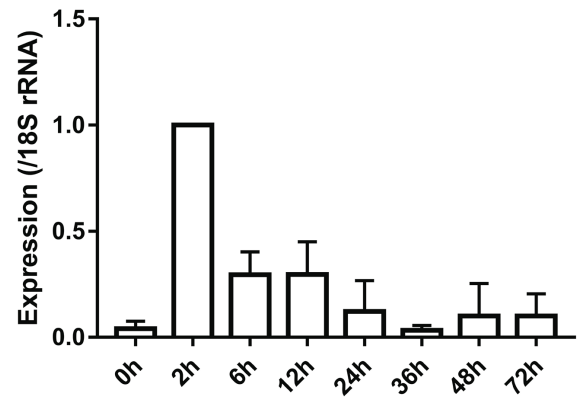

C

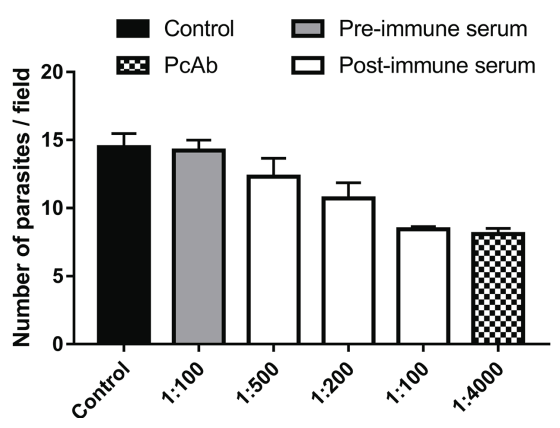

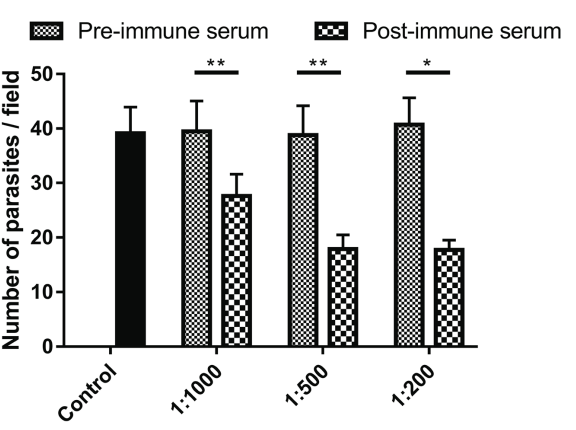
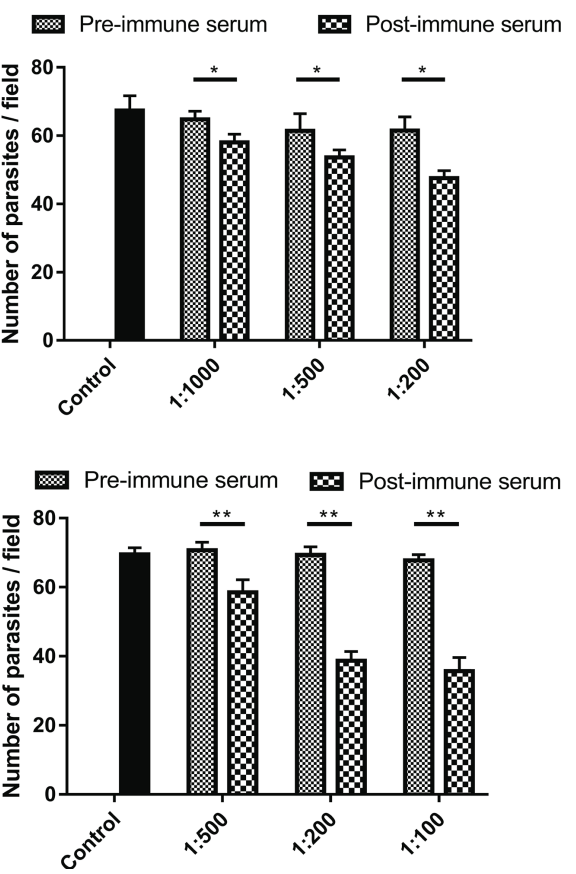

FIGURE 6 | Assessment of biological functions of MEDLE proteins. (A) Expression levels of the cgd5_4600 gene in developmental stages of C. parvum (left panel) and neutralization efficiency of immune sera against CPMEDLE-3 on C. parvum invasion (right panel). The expression level of the cgd5_4600 gene in C. parvum culture was determined by qPCR at various time points, with data being normalized with data from the expression of the Cp18S rRNA. Neutralization efficiency of post-immune sera against CPMEDLE-3 on C. parvum invasion was measured in HCT-8 cell culture. Data presented are mean \pm SD from three independent experiments for both the expression and neutralization studies. (B) Expression levels of the cgd6_5480 gene in developmental stages of C. parvum (left panel) and neutralization efficiency of immune sera against CPMEDLE-5 on C. parvum invasion (right panel). The expression level of the cgd6_5480 gene in C. parvum culture was determined by qPCR at various time points, with data being normalized with data from the expression of the Cp18S rRNA. Neutralization efficiency of immune sera against CpMEDLE-5 on C. parvum invasion was measured in HCT-8 cell culture. Data presented are mean \pm SD from three independent experiments for both the expression and neutralization studies. (C) Neutralization efficiency of polyclonal antibodies (PcAb) and immune sera against ChMEDLE-1 on C. hominis (left panel) and C. parvum (right panel) invasion. Cultures were treated with PcAb, immune sera, or pre-immune sera. Data presented are mean \pm SD from two (for $C$. hominis) or three (for $C$. parvum) independent experiments. The statistical significance of differences between treatment groups is indicated for neutralization studies of all three MEDLE proteins above the bars $\left({ }^{*} p<0.05 ;{ }^{* *} p<0.01\right)$.

proteins of C. parvum, results of the neutralization studies agree with the suggestion that MEDLE proteins may contribute to the host specificity of Cryptosporidium spp.

Although we have obtained some preliminary evidence to support the suggestion that MEDLE proteins are differentially expressed and play different roles in the Cryptosporidium lifecycle, more biologic and immunohistological studies, including the use of genetic manipulation of the pathogen by CRISPR/ Cas9, are needed to better understand the mechanism of MEDLE proteins during invasion of various Cryptosporidium spp. (Beverley, 2015). Considering the significant inhibition rates offered by their antibodies, MEDLE proteins could be potential targets in the design of new drugs or vaccines against cryptosporidiosis. The elucidation of crystal structures of MEDLE proteins would be a logic move toward this goal. These studies are likely leading to improved understanding of the invasion process of Cryptosporidium spp. and the development of new intervention strategies. 


\section{DATA AVAILABILITY}

All datasets generated for this study are included in the manuscript and/or the supplementary files.

\section{AUTHOR CONTRIBUTIONS}

YF and LX designed the study. JS, CJ, and JF performed the experiments. JS, YF, HW, NL, YG, and LX performed

\section{REFERENCES}

Abrahamsen, M. S., Templeton, T. J., Enomoto, S., Abrahante, J. E., Zhu, G., Lancto, C. A., et al. (2004). Complete genome sequence of the apicomplexan, Cryptosporidium parvum. Science 304, 441-445. doi: 10.1126/science.1094786

Arrowood, M. J., and Donaldson, K. (1996). Improved purification methods for calf-derived Cryptosporidium parvum oocysts using discontinuous sucrose and cesium chloride gradients. J. Eukaryot. Microbiol. 43:89S. doi: 10.1111/ j.1550-7408.1996.tb05015.x

Beverley, S. M. (2015). Parasitology: CRISPR for Cryptosporidium. Nature 523, 413-414. doi: 10.1038/nature14636

Bhalchandra, S., Cardenas, D., and Ward, H. D. (2018). Recent breakthroughs and ongoing limitations in Cryptosporidium research. F1000Res. 7:1380. doi: 10.12688/f1000research.15333.1

Cai, X., Woods, K. M., Upton, S. J., and Zhu, G. (2005). Application of quantitative real-time reverse transcription-PCR in assessing drug efficacy against the intracellular pathogen Cryptosporidium parvum in vitro. Antimicrob. Agents Chemother. 49, 4437-4442. doi: 10.1128/AAC.49.11.4437-4442.2005

Cevallos, A. M., Zhang, X., Waldor, M. K., Jaison, S., Zhou, X., Tzipori, S., et al. (2000). Molecular cloning and expression of a gene encoding Cryptosporidium parvum glycoproteins gp40 and gp15. Infect. Immun. 68, 4108-4116. doi: 10.1128/IAI.68.7.4108-4116.2000

Chalmers, R. M., and Davies, A. P. (2010). Minireview: clinical cryptosporidiosis. Exp. Parasitol. 124, 138-146. doi: 10.1016/j.exppara.2009.02.003

Checkley, W., White, A. C. Jr., Jaganath, D., Arrowood, M. J., Chalmers, R. M., Chen, X. M., et al. (2015). A review of the global burden, novel diagnostics, therapeutics, and vaccine targets for Cryptosporidium. Lancet Infect. Dis. 15, 85-94. doi: 10.1016/S1473-3099(14)70772-8

Efstratiou, A., Ongerth, J. E., and Karanis, P. (2017). Waterborne transmission of protozoan parasites: review of worldwide outbreaks-an update 2011-2016. Water Res. 114, 14-22. doi: 10.1016/j.watres.2017.01.036

Fei, J., Wu, H., Su, J., Jin, C., Li, N., Guo, Y., et al. (2018). Characterization of MEDLE-1, a protein in early development of Cryptosporidium parvum. Parasit. Vectors 11:312. doi: 10.1186/s13071-018-2889-2

Feng, Y., Li, N., Roellig, D. M., Kelley, A., Liu, G., Amer, S., et al. (2017). Comparative genomic analysis of the IId subtype family of Cryptosporidium parvum. Int. J. Parasitol. 47, 281-290. doi: 10.1016/j.ijpara.2016.12.002

Feng, Y., Ryan, U. M., and Xiao, L. (2018). Genetic diversity and population structure of Cryptosporidium. Trends Parasitol. 34, 997-1011. doi: 10.1016/j. pt.2018.07.009

Feng, Y., Tiao, N., Li, N., Hlavsa, M., and Xiao, L. (2014). Multilocus sequence typing of an emerging Cryptosporidium hominis subtype in the United States. J. Clin. Microbiol. 52, 524-530. doi: 10.1128/JCM.02973-13

Gubbels, M. J., and Duraisingh, M. T. (2012). Evolution of apicomplexan secretory organelles. Int. J. Parasitol. 42, 1071-1081. doi: 10.1016/j. ijpara.2012.09.009

Guo, Y., Tang, K., Rowe, L. A., Li, N., Roellig, D. M., Knipe, K., et al. (2015). Comparative genomic analysis reveals occurrence of genetic recombination in virulent Cryptosporidium hominis subtypes and telomeric gene duplications in Cryptosporidium parvum. BMC Genomics 16:320. doi: 10.1186/s12864-015-1517-1 the statistical analysis and interpreted the results. JS, YF, and LX developed the draft manuscript. All authors contributed to manuscript revisions and approved the final version for publication.

\section{FUNDING}

This work was supported by the National Natural Science Foundation of China (31630078 and 31425025).

Jakobi, V., and Petry, F. (2006). Differential expression of Cryptosporidium parvum genes encoding sporozoite surface antigens in infected HCT-8 host cells. Microbes Infect. 8, 2186-2194. doi: 10.1016/j.micinf.2006.04.012

Kuhlenschmidt, T. B., Rutaganira, F. U., Long, S., Tang, K., Shokat, K. M., Kuhlenschmidt, M. S., et al. (2016). Inhibition of Calcium-dependent protein kinase 1 (CDPK1) in vitro by pyrazolopyrimidine derivatives does not correlate with sensitivity of cryptosporidium parvum growth in cell culture. Antimicrob. Agents Chemother. 60, 570-579. doi: 10.1128/AAC.01915-15

Li, B., Wu, H., Li, N., Su, J., Jia, R., Jiang, J., et al. (2017). Preliminary characterization of MEDLE-2, a protein potentially involved in the invasion of Cryptosporidium parvum. Front. Microbiol. 8:1647. doi: 10.3389/ fmicb.2017.01647

Liu, S., Roellig, D. M., Guo, Y., Li, N., Frace, M. A., Tang, K., et al. (2016). Evolution of mitosome metabolism and invasion-related proteins in Cryptosporidium. BMC Genomics 17:1006. doi: 10.1186/s12864-016-3343-5

Livak, K. J., and Schmittgen, T. D. (2001). Analysis of relative gene expression data using real-time quantitative PCR and the 2(-Delta Delta C(T)) Method. Methods 25, 402-408. doi: 10.1006/meth.2001.1262

Matsubayashi, M., Teramoto-Kimata, I., Uni, S., Lillehoj, H. S., Matsuda, H., Furuya, M., et al. (2013). Elongation factor-1alpha is a novel protein associated with host cell invasion and a potential protective antigen of Cryptosporidium parvum. J. Biol. Chem. 288, 34111-34120. doi: 10.1074/jbc.M113.515544

Mauzy, M. J., Enomoto, S., Lancto, C. A., Abrahamsen, M. S., and Rutherford, M. S. (2012). The Cryptosporidium parvum transcriptome during in vitro development. PLoS One 7:e31715. doi: 10.1371/journal.pone.0031715

Murugaiyan, J., and Roesler, U. (2017). MALDI-TOF MS profiling-advances in species identification of pests, parasites, and vectors. Front. Cell. Infect. Microbiol. 7:184. doi: 10.3389/fcimb.2017.00184

Ryan, U., and Hijjawi, N. (2015). New developments in Cryptosporidium research. Int. J. Parasitol. 45, 367-373. doi: 10.1016/j.ijpara.2015.01.009

Singh, P., Mirdha, B. R., Srinivasan, A., Rukmangadachar, L. A., Singh, S., Sharma, P., et al. (2015). Identification of invasion proteins of Cryptosporidium parvum. World J. Microbiol. Biotechnol. 31, 1923-1934. doi: 10.1007/ s11274-015-1936-9

Upton, S. J., Tilley, M., and Brillhart, D. B. (1995). Effects of select medium supplements on in vitro development of Cryptosporidium parvum in HCT-8 cells. J. Clin. Microbiol. 33, 371-375.

Xiao, L. (2010). Molecular epidemiology of cryptosporidiosis: an update. Exp. Parasitol. 124, 80-89. doi: 10.1016/j.exppara.2009.03.018

Conflict of Interest Statement: The authors declare that the research was conducted in the absence of any commercial or financial relationships that could be construed as a potential conflict of interest.

Copyright (C) $2019 \mathrm{Su}$, Jin, Wu, Fei, Li, Guo, Feng and Xiao. This is an open-access article distributed under the terms of the Creative Commons Attribution License (CC BY). The use, distribution or reproduction in other forums is permitted, provided the original author(s) and the copyright owner(s) are credited and that the original publication in this journal is cited, in accordance with accepted academic practice. No use, distribution or reproduction is permitted which does not comply with these terms. 